\title{
The Evolution of NIMHANS Neurotrauma Care
}

\author{
Dhananjaya I. Bhat ${ }^{1} \quad$ B. Indira Devi ${ }^{2}$ Dhaval Shukla ${ }^{2}$ Akshay V. Kulkarni² \\ ${ }^{1}$ Department of Neurosurgery, RV Aster Hospital, Bangalore, \\ Karnataka, India \\ 2Department of Neurosurgery, National Institute of Mental Health \\ and Neuro-Sciences (NIMHANS), Bangalore, Karnataka, India

\begin{abstract}
Address for correspondence B. Indira Devi, MCh, Department of Neurosurgery, National Institute of Mental Health and NeuroSciences (NIMHANS), Bangalore 560029, Karnataka, India (e-mail: bidevidr@gmail.com).
\end{abstract}

Indian J Neurotrauma:2020;17:62-68

\begin{abstract}
Keywords

- NIMHANS

- history of neurotrauma

NIMHANS

- lean sigma

- neurotrauma

management in India

- neurotrauma tam

Neurotrauma services in NIMHANS, Bangalore, have been evolving since its inception in 1958. At present, it is a major referral center for Karnataka and surrounding districts of the southern states. It has many firsts to its credit, from having a separate building to having dedicated trauma teams to manage the service $24 / 7$. It has made rapid strides in healthcare delivery based on data-driven evidence, improvisation with Lean sigma approach, and contribution through research and publications. This center has won best Neurotrauma center award in 2010.
\end{abstract}

\section{Introduction}

The National Institute of Mental Health and Neurosciences (NIMHANS), Bengaluru, is a multidisciplinary institution, providing services in neurology, neurosurgery and psychiatry.

The journey of this institute started as a lunatic asylum in 1848. Subsequently, it was renamed as Mysore government mental hospital in 1926 and later, the All India Institute of Mental Health in 1954. Prof. M. V. Govindswamy, a psychiatrist and the founding director, was a great visionary. He proposed and materialized the idea of all neurosciences being together. Dr. Govindswamy began performing neurosurgeries and later, Dr. R. M. Varma joined as the first neurosurgeon at this institute. In 1958, under the auspices of these two giants, the department of neurosurgery was established and Dr. Varma became the head of the department. Later in 1975, the institute was renamed as NIMHANS. The details of the history of the neurosurgery department is available in Neurology India, January 2015.

Initially, neurosurgery patients were admitted to psychiatry wards and operated in the wings of the ward. The anesthetic from Victoria hospital used to help out in addition to his work there.

The evolution of the neurotrauma services can be divided into major landmark time periods (-Fig. 1).
The first trauma-related surgery to be performed in NIMHANS was a chronic subdural hematoma on January 7, 1958. A full-time anesthetist was appointed in 1962.

In 1963, along with Dr. G.N. Reddy, Dr. Varma proposed the construction of a separate building, a neurocenter, for neurological and neurosurgical patients. The neurocenter was inaugurated on December 30, 1973.

At its inauguration, the neurocenter had 80 beds, two operation theaters, a blood bank, and state-of-the-art investigative laboratories. On December 27, 1974, NIMHANS was born, with Dr. R.M Varma and Dr. G.N. Reddy leading the department. Having recognized the need for dedicated head injury care, a 5-bed head injury ward was commissioned/ became operational in 1977. District surgeons were trained over the course of a 7-day module, both in basic and practical instruction, with a steady growth recorded in the department. Neurotrauma had witnessed rapid referral patterns of care $^{1}$ over decades of neurotrauma care. From its inception in 1958, the department has gradually taken the role of leading center for Karnataka and nearby states in the neurotrauma services (- Table $\mathbf{1}$ ).

Posttrauma clinic: In 1979, a specialized posttrauma clinic was commissioned with a neurosurgeon, a neurologist, and a psychiatrist for the head-injury patients. This offers a
DOI https://doi.org/

$10.1055 / \mathrm{s}-0040-1713313$

ISSN 0973-0508.
License terms

() (1) $\ominus \circledast$ 


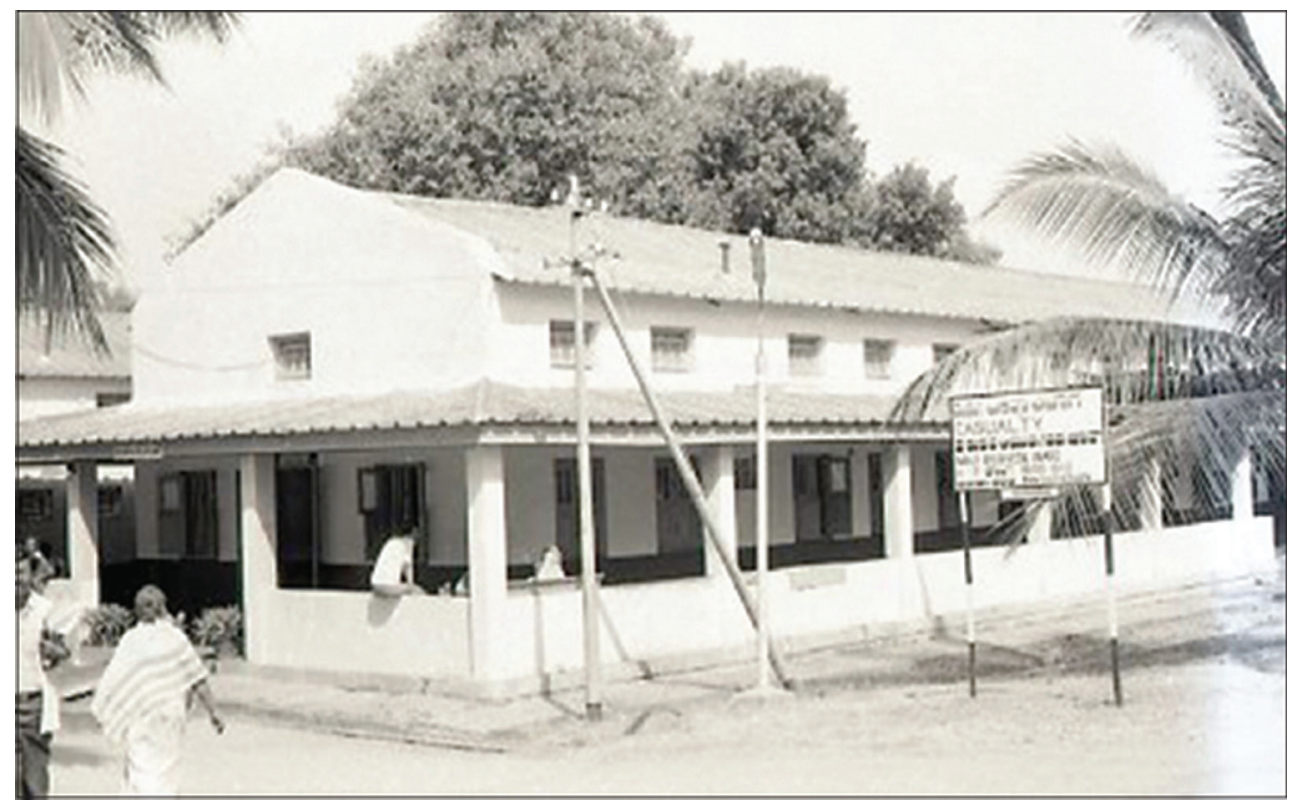

Fig. 1 Casualty in 1970-The Beginning. From inception to the commissioning of the neurocenter.

Table 1 The number of patients operated is detailed below

\begin{tabular}{|l|l|l|l|}
\hline S/N & Year & Total & $\begin{array}{l}\text { Trauma } \\
\text { (Head + spinal) }\end{array}$ \\
\hline 1 & 1958 & 15 & - \\
\hline 3 & $1960-61$ & 132 & 39 \\
\hline 13 & $1970-71$ & 452 & \\
\hline 14 & $1980-81$ & 1336 & 235 \\
\hline 24 & $1990-91$ & 2468 & 582 \\
\hline 34 & $2000-01$ & 2790 & 620 \\
\hline 44 & $2010-11$ & 5710 & 1276 \\
\hline 47 & $2013-14$ & 6390 & 1484 \\
\hline 50 & $2016-17$ & 8369 & 2019 \\
\hline 51 & $2017-18$ & 7724 & 1879 \\
\hline
\end{tabular}

comprehensive psychiatric and neurosurgical care, including rehabilitation for patients with history of trauma.

\section{The 80s}

The department of neurosurgery in collaboration with epidemiology had produced a large number of papers and big data was generated. Based on these, many policy changes/ decisions were taken. One of the prime examples is objective criteria about the morbidity and mortality rates of 2-wheeler and pillion riders when helmet is compulsory and when it was not compulsory (Yadukul). ${ }^{2}$

NIMHANS has been recognized as a WHO collaborating center for trauma (generally $40 \%$ of trauma is neurotrauma). Prof. Gururaj G. has been a passionate and active investigator of trauma and road traffic accidents of Bangalore in particular and Karnataka in general. His recent paper in Lancet deals with statistical analysis of mortality due to road accidents in Indian states.

Under the guidance of Prof BS Das, the department steadily grew and consolidated its experience in neurotrauma.

\section{The 90s}

NIMHANS (Department of Neurosurgery) steadily gained expertise with regard to the management of neurotrauma-related head injuries, spine injuries, and peripheral nerve injuries. Prof. B.S. Das, who headed the department from 1981 to1996, was engaged in conducting complex craniovertebral (CV) junction injuries and peripheral nerve injuries. He popularized transoral odontoidectomy and indigenously developed the contoured rod for occipito-cervical fixation. He was also actively involved in popularizing surgery for brachial plexus injuries. During this time, emergency services were offered in the ground floor of the outpatient department (OPD) complex.

Some degree of space organization for triage, resuscitation, intensive observation recovery room and ICU, short stay wards, CT scan, and X-ray services were available. Two operation theaters were also available. The advantage of the arrangement was, with team doctors available in the OPD in the first floor, that quick mobilization of extra hands could be organized.

There have been continuous efforts to improve the patient care and create patient friendly delivery of neurotrauma care.

- Manpower development.

- Data driven protocols.

- Infrastructure development.

- Evidence based medicine.

\section{Manpower Development}

In 2002, there was a major change in trauma center protocols. Previously, the trauma was managed for 24 hours by the unit that handles OPD/emergency for the day. This is still the protocol at most of the centers across all the clinical specialty in India and abroad. At NIMHANS in 2002, two separate teams were commissioned for handling neurotrauma cases. 
This decision was taken by the department of neurosurgery, which was then headed by Prof KVR Sastry who supported the outgoing director Prof M. Gourie Devi and incoming director Prof D.N. Nagaraja.

Initially, the duty team (one of the three units) used to provide neurotrauma care for 24 hours by rotation. However, in 2002, the concept of a separate Trauma team was thought of. It was proposed that the trauma team would work two shifts, which would not only make it more efficient but also more productive and patient friendly. These two teams were designated as team one/morning team and team two/night team, which comprised 4 to 6 residents headed by the trauma chief resident. The teams were to be guided by the on-call consultant of the respective units. The trauma team also has a 10-bedded neurosurgical intensive care unit which is managed by them. The teams work for 12 hours each, 6 am to $6 \mathrm{pm}$ and vice versa, with handing over of all patient-related information and plans to each other. These teams work continuously for a month with the 2nd and 4th Sundays as relief, when unit residents take care of managing the shift change from day to night and vice versa for a period of 12 hours. At the end of the month, the residents are rotated to ward duties. During this entire month, residents take focused care of trauma and emergency admissions only. On an average, each resident gets a casualty posting every $3 \mathrm{rd}$ month. The team consists of a resident in each year-1st, 3rd, 4th, 5th, and final.

This concept of a separate trauma team is unique and NIMHANS, Bengaluru, is one of the first centers to have this facility.

\section{Infrastructure: Neurotrauma Center}

August 2008: A Separate building for neurotrauma and neurological emergencies was commissioned by another visionary director Prof Dr D.N. Nagaraja.
Infrastructure: This is a three-story structure with a large trauma bay which can accommodate up to 50 patients at a time. All these patients are on mobile trolley beds for fast mobilization to and from CT/X-ray/procedure rooms. Patients are kept here for short periods till evaluation and further planning is carried out. Patients are arranged as per triage in different rows. The trauma center also houses the following wards: Priority 1 ( 6 beds), Priority 2 which has 23 beds, postop recovery/short stay observation ward (20 beds), and a distantly located head injury ward ( 9 beds). The center has three dedicated OTs for trauma with microscopes and other fully functional equipment. There are two $\mathrm{CT}$ machines and one X-ray machine dedicated for the center which are placed within 20 to $30 \mathrm{~m}$ distance from the neurosurgery desk for faster evaluation. Designated pathology and microbiology laboratories are available 24 hours.

This center and its unique methodology have been running successfully since then. Many different medical colleges from across the state send their postgraduate residents in neurosurgery (MCh and DNB), OMFS, ENT, and orthopedics for experience in polytrauma and neurosurgical

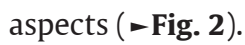

\section{Lean Sigma Team Assessment of Emergency Services}

Strides: Lean Sigma approach.

The Lean Six Sigma is an approach designed to improvise the quality of a process/system by analyzing and eliminating waste points and loopholes of resources. This approach is designed to expose the sources of inefficiency in a workflow to maintain a steady flow of work process. The approach reduces the variables and standardizes the results. The Lean Six Sigma approach defines the following seven forms of waste: motion (whether the patient is moving within hospital

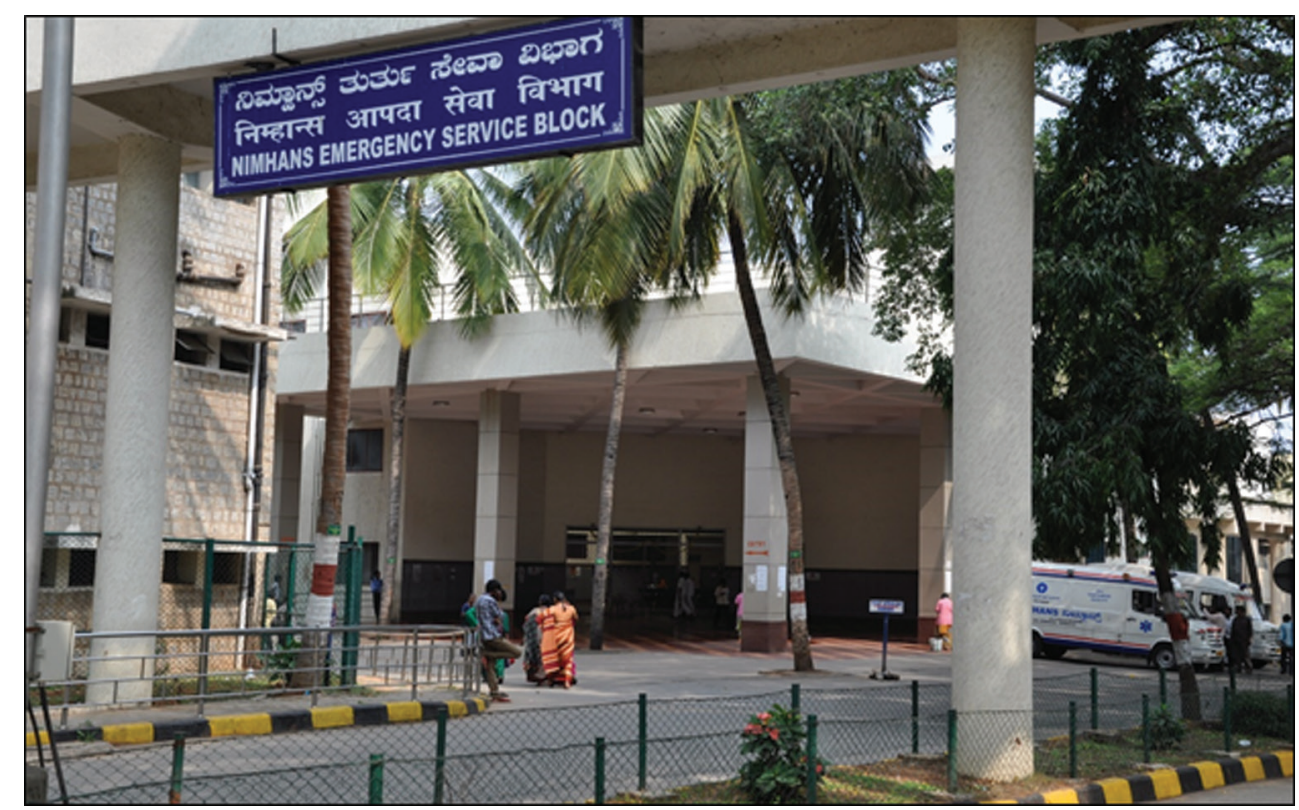

Fig. 2 NIMHANS Casualty (Golden Jubilee Complex). 
facility for services effectively?), inventory (all equipment and services available at a single station in proximity?), waiting time (waiting time for patients to receive a service at one station?), defects (are all required/available equipment functional?), overproduction (protocols efficiency in operative decision-making?), transportation (emergency ambulatory transportation availability, connecting road quality, traffic, and parking?), and over processing (patients undergoing unnecessary scans?). A team from Lean Six Sigma assisted us in identifying all the possible blockades in managing patients in the hospital setup while evaluating patients with identical characteristics, referral patterns, and provided pre-hospital care.

The team identified the following blockades at NIMHANS:

- The wait time at the triage area for evaluating the neurosurgery resident at emergency services.

- The wait time for the radiological investigations is longer than expected.

- The wait time for the consultation with the senior consultants before making a patient management plan.

- Overburdening of the tertiary/specialized healthcare center by unnecessary referral of mild TBI patients.

- Time spent in transportation.

- Unaccounted time in treatment and discharge.

The care provided by NIMHANS neurotrauma center has undergone further improvements since then.

\section{Improvement of Prehospital Neurotrauma Care Delivery}

\section{8- National Ambulance Services}

The 108 Emergency Medical Service provides first aid as well as basic life support and advanced life support. These ambulances have fully trained paramedics onboard to reach site in 20 minutes on an average and provide resuscitation and emergency medical aid. Once prehospital aid is taken care of, the patient is transported to the nearest trauma center/hospital. The 108 emergency service teleoperators also provide facilities to contact doctor or police, if needed. This facility has improvised patient transport from all around Bengaluru and nearby places. NIMHANS facilities can outreach a greater number of patients.

In addition, 108 services NIMHANS has ambulances of its own, with emergency care support, which were donated by the State Bank of India ( - Fig. 3 ).

At present, six units of neurosurgery handle the neurosurgery OPD of the institution. Sixteen senior consultants and professors operate in six units, with 43 residents working in rotations to handle OPD, emergency Cases, and inpatients.

\section{Workspace Organization}

After being attended to by a casualty medical officer, the patient receives TT; thereafter, resuscitation is started and neurosurgical referral is given. All this is organized in the triage area $(\boldsymbol{- F i g .} \mathbf{3})$. After being assessed by a neurosurgery resident, the patient may undergo a CT scan, X-ray of chest, etc., all of which are located right next to the casualty/ triage hall. Once the scan is conducted, either the patient is scheduled for surgery or kept for further observation in either the Priority 1 (requiring intense monitoring) or Priority 2 wards (-Fig. 4, - Fig. 5A \& 5B).

There are three operation theaters available in the same building complex provided with state-of-the-art monitors and operation facilities, ably supported by neuroanesthetists ( - Fig. 6A \& 6B).

Following surgery, patients are observed in the recovery room and kept for 24 hours in the short stay ward ( - Fig. 7). There is a dedicated head injury ward with 9 beds and a

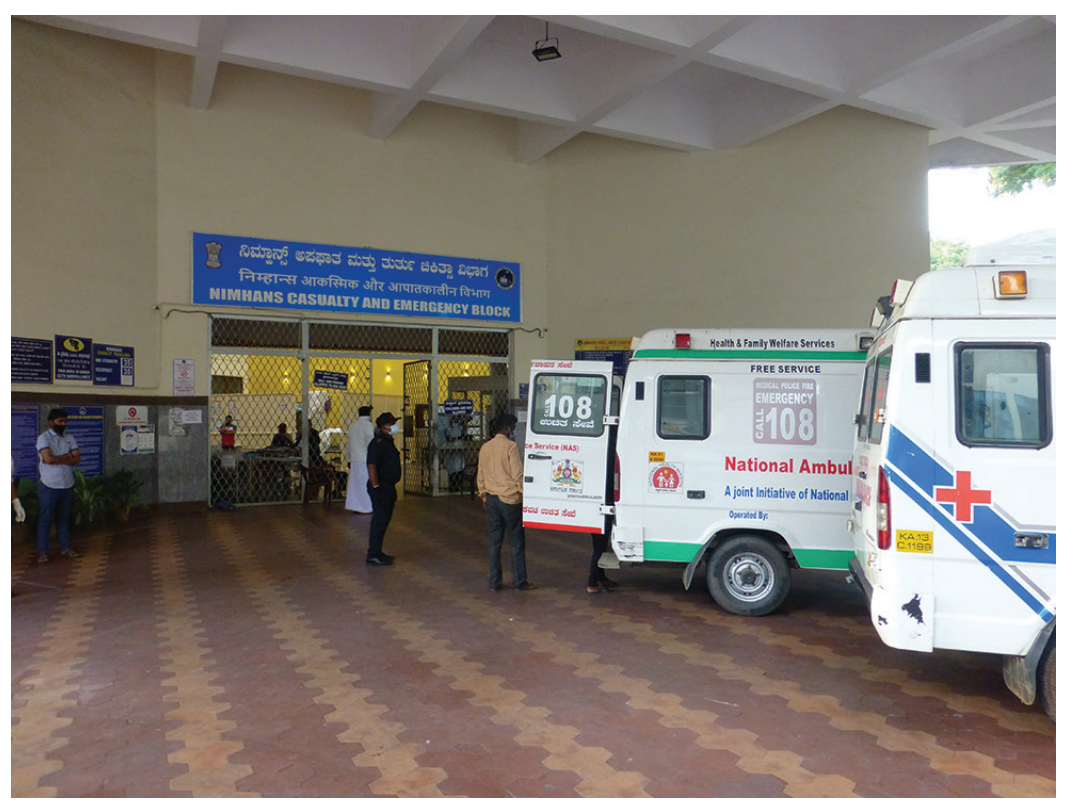

Fig. 3 Ambulance bay/patient receiving area. 


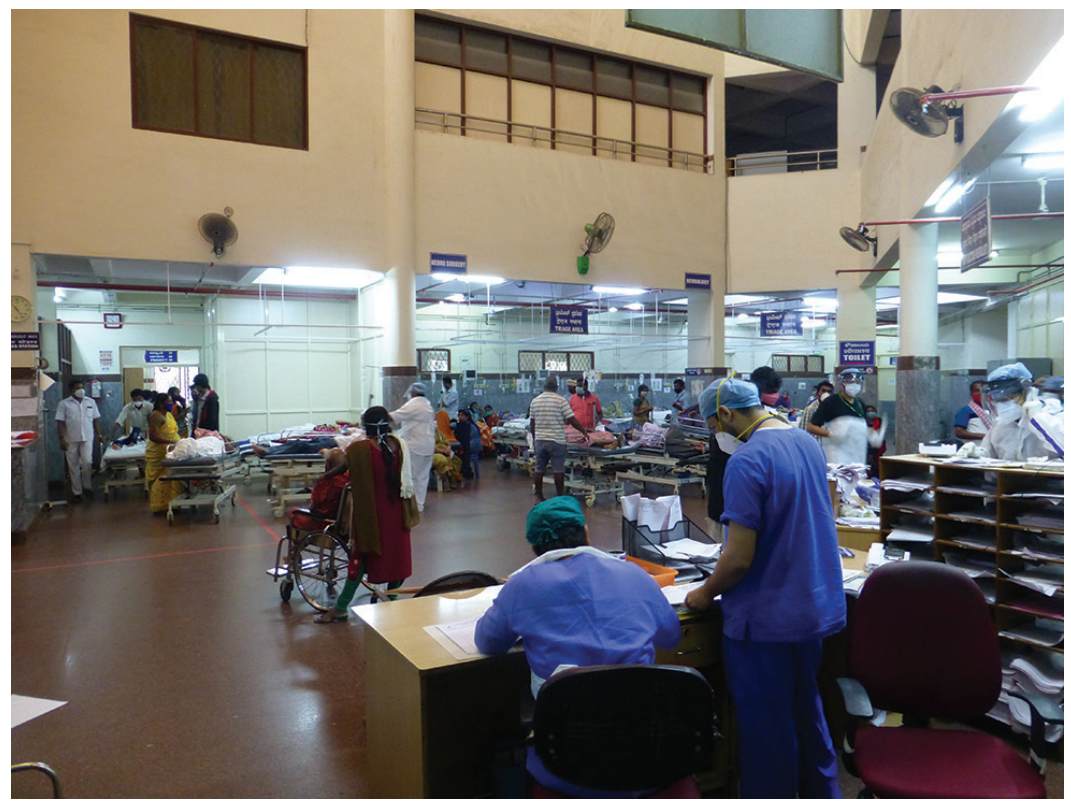

Fig. 4 Casualty inside view (Triage area).
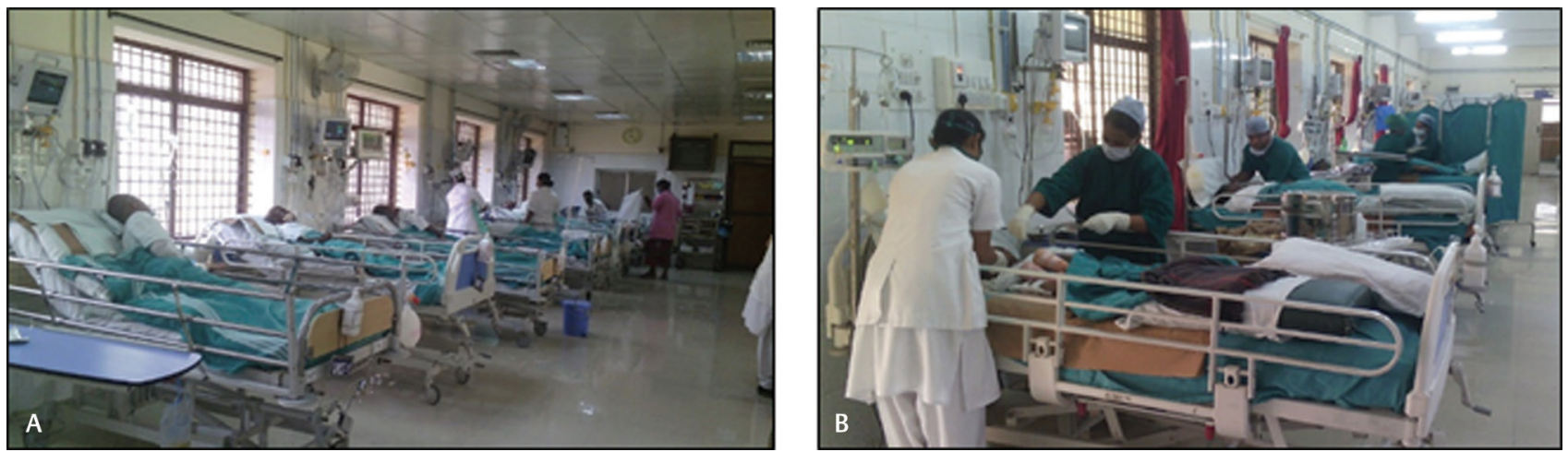

Fig. 5 (A) Priority 1 ward. (B) Priority 2 ward.
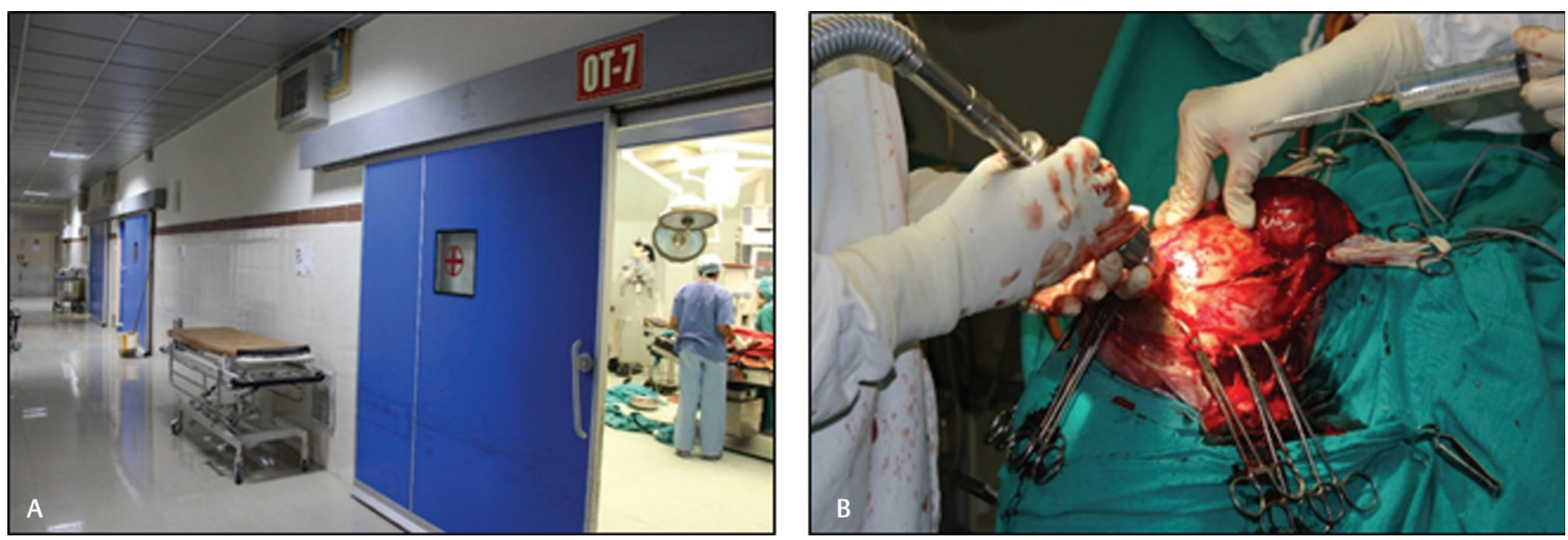

Fig. 6 (A) Two operation theaters $\times 24$ hours. (B) Inside OT room (ongoing surgery). 


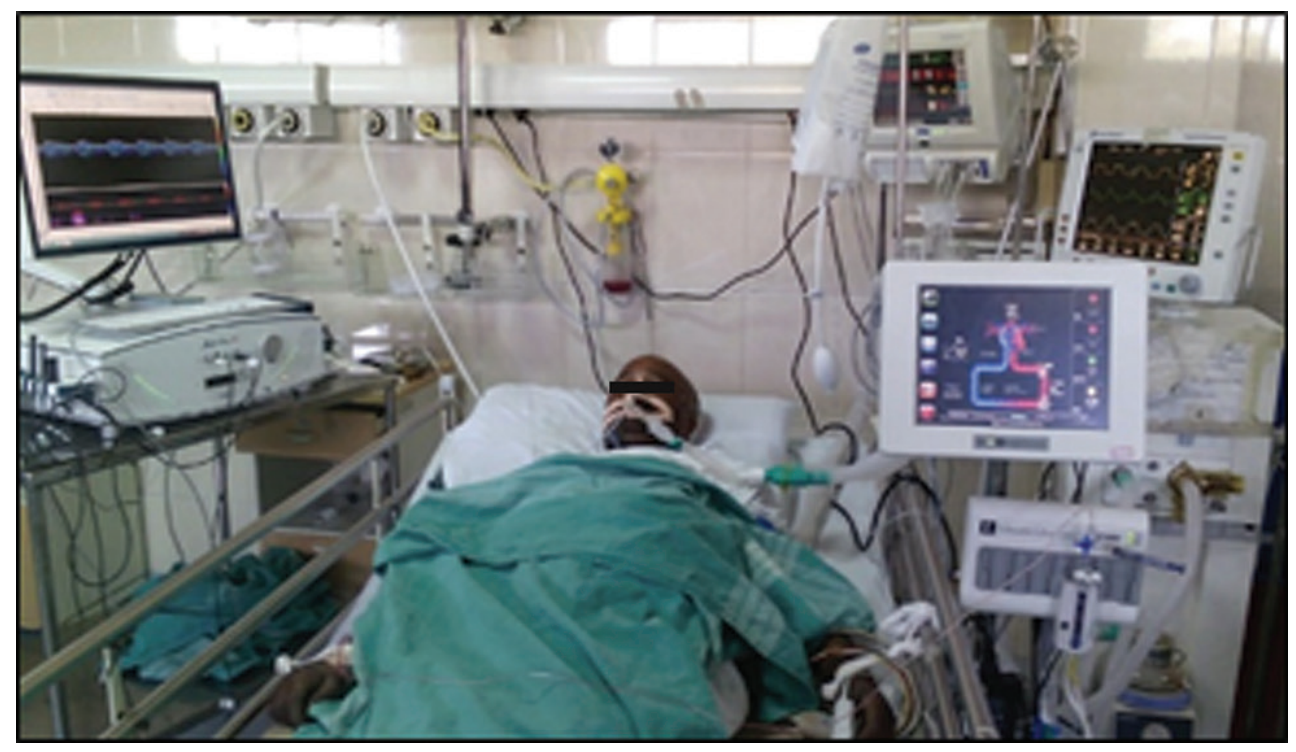

Fig. 7 ICU.

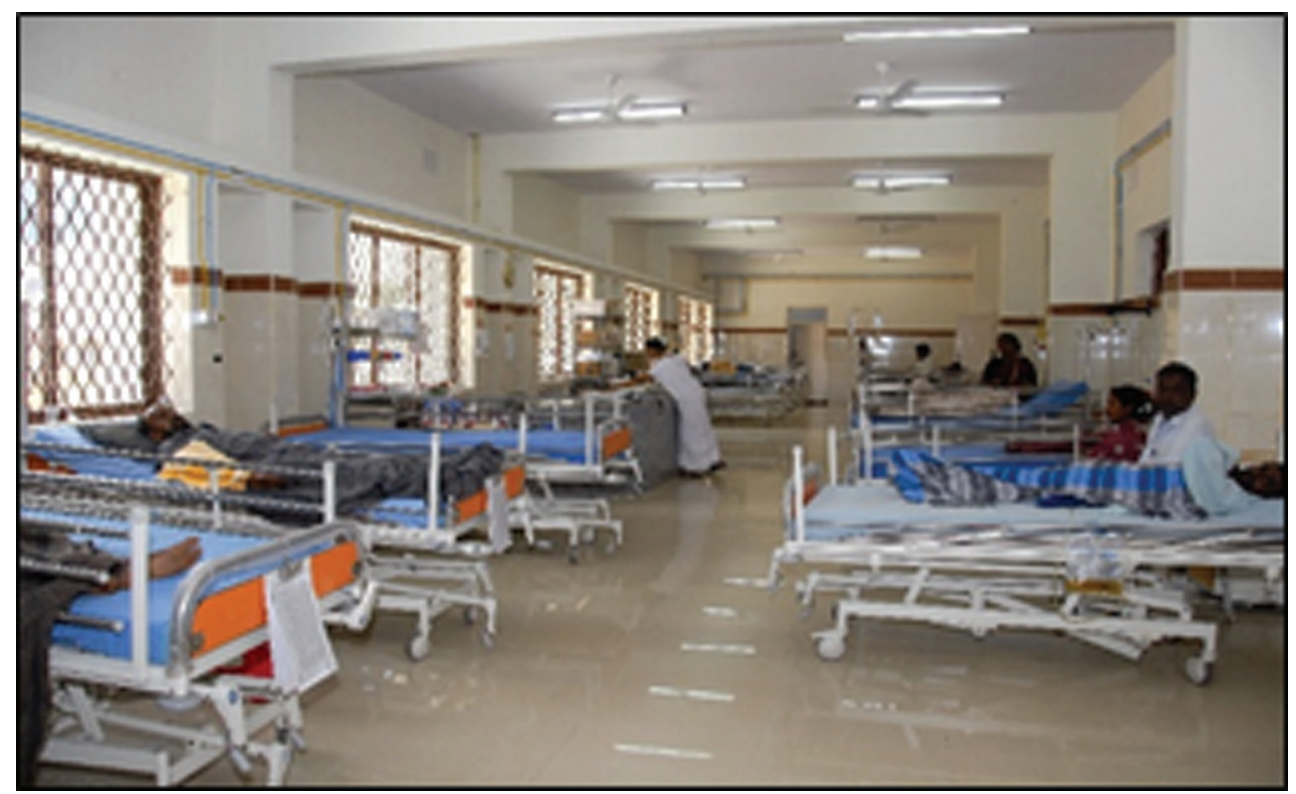

Fig. 8 Patients under observation.

6-bed stepdown ward for patients requiring stepdown care after ICU stay ( - Fig. 8).

Further rehabilitation is available in a separate building where patients may utilize either inpatient or outpatient rehabilitation. The payment modalities for services offered differ from free of cost for unknown patients brought by 108 or BPL patients to payment for APL.

\section{Laurels}

NTSI annual conference 2010 awarded the best neurotrauma service accolade to this dedicated trauma center.

NIMHANS conducted the 24th Annual conference of Neurotrauma Society of India in August 2015. The eminent orators were Dr. David Mendelow, NewCastle upon Tyne, Dr. Peter Hutchinson, Addenbrook Hospital Cambridge, UK, and Prof Shobini Rao, former Professor of Neupsychology, NIMHANS. This conference was well-attended and was appreciated for its scientific content and presentation.

Since 2015, the neurotrauma center has incorporated Lean sigma approach.

The department has always been a big center for data collection of protocols/studies, the department which has participated in various international clinical trials and successfully completed recruiting for Rescue SDH and GNOS. This has been a nodal center for research and pioneering ideas of neurotrauma in India. The center was one of the finalists of 2015 BMJ South East Asia Award for Best Trauma center. The center has participated in the consensus statement for Decompressive Craniectomy, Cambridge, September 2017. The center has actively participated in generating TBI Guidelines for India. 
NIMHANS has more than 100 indexed publications for neurotrauma.

NIMHANS is going to host the prestigious ICRANNTSI-2020 in August 2020.

\section{Neurosurgical Care Delivery, Future Plans}

The condition of neurotrauma care delivery can be smoothened by improving the primary and the secondary healthcare facilities, in order to reduce the referral of unnecessary patients and subsequently overburdening of the tertiary healthcare facility. The approach of Internet and telemedicine in managing patients of head trauma at remote facilities can be efficient by reducing the burden of specialized healthcare centers.

- There should be a separate department of a telemedicine equipped with Internet connectivity at each level facility, every district hospital, medical college hospitals and tertiary healthcare facility/specialized facility, with 24/7 operator personnel who are connected with on-duty neurosurgeons.
- The primary CT scans and radiological findings should be shared on the Internet portal to the secondary/tertiary healthcare facility for analysis before referring and overburdening a secondary or a tertiary healthcare system.

- The emergency medical response should be trained to evaluate a patient for head injuries. On primary evaluation, the emergency response team can contact a telemedicine team at a specialized health center.

- The emergency response team should provide prehospital care to the patient and transport them to the nearest healthcare facility which are able to carry treatment for them.

\section{Conflict of Interest}

None declared.

\section{References}

1 Bhat DI, Devi IB. History of neurosurgery at National Institute of Mental Health and Neurosciences: an epitome of steady growth. Neurol India 2015;63(1):91-95

2 Yadukul S, Devadass PK, Gururaj G. Role of Helmet in Preventing Head Injury among Two Wheeler Occupants in Fatal Road Traffic Injuries. Indian Journal of Forensic Medicine \& Toxicology 2016. Doi:10. 6. 10.5958/0973-9130.2016.00002.5 